\title{
Vom Livestream bis zur Gassenfeier. Vielfältige Bilder gottesdienstlicher Feiern der ersten 18 Monate der Pandemie
}

Saskia Löser

\section{Einleitung}

„Freude und Hoffnung, Trauer und Angst der Menschen von heute, besonders der Armen und Bedrängten aller Art, sind auch Freude und Hoffnung, Trauer und Angst der Jünger Christi. Und es gibt nichts wahrhaft Menschliches, das nicht in ibren Herzen seinen Widerhall fände." (GS 1)

Diese ersten Sätze aus der Pastoralkonstitution des Zweiten Vatikanischen Konzils (1962-1965) Gaudium et Spes (GS) bilden den Ausgangspunkt für die hier vorliegenden Überlegungen zu den gottesdienstlichen Feiern im Kontext der Corona-Pandemie. Diese Worte sind auch Grundlage für die Feier von Gottesdiensten überhaupt. Im Gottesdienst kommen Menschen zusammen und bringen das, was sie bewegt und beschäftigt, in Lob und Dank, Bitte und Klage vor Gott und voreinander. Das erste Jahr der Covid-19-Pandemie war liturgisch geprägt von unterschiedlichen Verschärfungen und Lockerungen - wie dies auch in den Bereichen des öffentlichen Lebens, der Kultur und Gastronomie und im privaten Leben der Fall war.

Es ist eine Zeit, in der Vertrautes Halt gab, verändert und neu entdeckt wurde. Es ist eine Zeit der Aufbrüche und Kreativität auf der einen Seite - auf der anderen stehen Schmerzen und Ängste. Die Einschränkungen betreffen alle Lebensbereiche und alle Menschen, wenn auch in unterschiedlichem Ausmaß. Die Ängste berühren die Existenz der eigenen Person. Unsicherheit herrscht auch in der Suche nach Antworten auf die Frage nach der Relevanz der Pfarrgemeinden und Kirchen. Es ist ihr hoher Anspruch, in "Trauer und Angst“ für die Menschen Ansprechpartnerin zu sein. Diesem Anspruch versuchten viele haupt- und ehrenamtliche Mitarbeiter*innen der Kirche ${ }^{1}$ gerecht zu werden, indem sie sich vielfach

1 Ich beziehe mich in meinen Ausführungen auf die römisch-katholische Kirche im deutschsprachigen Raum. Paralleles lässt sich vermutlich auch über andere 
darum bemühten, kirchliches Leben in den Vollzügen der Liturgie, Gemeinschaft, Diakonie und des Glaubenszeugnisses aufrecht zu erhalten. Nicht allen war dies möglich. Andere verwirklichten neue Ideen, nutzen vorhandene Ressourcen und entwickelten diese weiter. Im Bereich der Technik und der digitalen Möglichkeiten kam es zu einer Professionalisierung, die besonders in den gottesdienstlichen Feiern zu spüren ist.

Anhand von sieben Bildern persönlicher Erfahrungen dieser letzten Monate wird in diesem Beitrag ein Einblick (der nicht den Anspruch auf Vollständigkeit erheben kann) in das gottesdienstliche Leben der ersten 18 Monate der Corona-Pandemie gegeben. Es ist eine erfahrungsbezogene Darstellung verbunden mit der Reflexion liturgiewissenschaftlicher Fragen, die sich insbesondere auf die Möglichkeiten der Partizipation, auf die Körperlichkeit und die Chancen und Grenzen der Liturgie in diesen Tagen beziehen. Es werden hier unterschiedliche gottesdienstliche Feiern vorgestellt - der Blick jedoch nicht auf Sakramente und Sakramentalien wie Hochzeiten, Beerdigungen, Taufen, Segnungen etc. gelegt. Einer der groBen Schmerzen dieser Zeit war es, dass Zusammenkünfte mit traurigem oder frohem Anlass überhaupt nicht oder nur eingeschränkt stattfinden konnten. Man kann jedoch von den dargestellten Feiern zu diesen hin Parallelen ziehen - wurden diese Feiern doch auch unter den geltenden Bestimmungen und in der kreativen Vielfalt der Möglichkeiten gefeiert. Taufen wurden beispielsweise analog gefeiert und gleichzeitig live für daheim gebliebene Angehörige und Bekannte gestreamt. Ebensolches gilt für Verabschiedungen.

\section{Einblicke}

Aus unzähligen möglichen Beispielen habe ich hier sieben Bilder ausgewählt, die einen Einblick geben in die Vielfalt der gefeierten Gottesdienste. Die Bilder basieren auf meinen Erlebnissen. Gleichzeitig weiß ich aus kollegialem und freundschaftlichem Austausch von ähnlichen Erfahrungen und Beobachtungen anderer Menschen.

Die sieben Feiern sind aus der großen Bandbreite von Online- und Offline-Formaten ausgewählt. Zunächst wird ein Einblick in drei analoge Feierformen gegeben. Hierfür wurden sowohl Feiern ausgewählt, bei denen eine Person zwar allein ist, jedoch in spiritueller Gemeinschaft, und Feiern

Kirchen und Glaubensgemeinschaften bzw. die römisch-katholische Kirche in anderen Ländern sagen. 
in räumlicher Gemeinschaft. Sodann werden vier digitale Feierformate angesprochen. Dabei wird deutlich, dass auch diese Feierformate nicht neu und keine Erfindungen der letzten Monate sind. Intensiviert hat sich jedoch die Anteilnahme, verändert haben sich die Gründe der Teilnahme an diesen Feiern.

\subsection{Analoge Gottesdienste: Hausgottesdienste und Messen mit Abstand}

„So entstand - fast in einem Nebensatz - die Idee, eine Kerzenaktion zu initiieren."

Am 16. März 2020 traf sich das Pastoralteam der Pfarrei St. Pankratius in Oberhausen-Osterfeld, um zu besprechen, welche Auswirkungen die gesetzlichen Maßnahmen zur Verhinderung der Ausbreitung von Covid-19 auf das Gemeindeleben haben wird, was nun zu tun sei und wie man den Mitgliedern der Pfarrgemeinde „Mut machen“3 könnte. Die dieser Besprechung entsprossene "Gebetseinladung, immer um 19.00 Uhr eine brennende Kerze ins Fenster zu stellen und ein Vaterunser zu beten bzw. leicht abgewandelt Stille zu halten" 4 verbreitete sich durch Bekanntmachung in unterschiedlichen Medien über das Gebiet der Gemeinde hinaus bis nach Südafrika und Israel. Diese Kerze im Fenster konnte ein Zeichen der Verbundenheit und der Hoffnung für Menschen unterschiedlicher religiöser Zugehörigkeit und auch für Atheist"innen sein.

So wie das Pastoralteam in Oberhausen-Osterfeld trafen sich vielerorts Pastoral- und andere Teams, um über Initiativen für die Gestaltung des Gebets- und Glaubensleben in dieser Zeit zu beratschlagen. Kreative Wege der Seelsorge wurden gesucht und in unterschiedlichen Ideen gefunden. So entstanden Feierhefte und andere Gebetsblätter, die zum Mitnehmen in Kirchen gelegt wurden. In Weihwasserbecken konnte man in kleine Fläschlein abgefülltes Weihwasser finden und am Palmsonntag Palmzweige abholen - entweder schon zuvor vom Priester gesegnete oder in Kombination mit einem Segensgebet, welches selbst gesprochen werden konnte. In Kirchenzeitungen wurden vermehrt Vorschläge zur Gestaltung des Sonntags abgedruckt. Nach der ersten Zeit des strengen Lockdowns wurden thematische Stationen in Kirchen aufgebaut, die zur Einkehr und Reflexion einluden.

2 Wichmann, Das \#hoffnungslicht aus Osterfeld, 425.

3 Wichmann, Das \#hoffnungslicht aus Osterfeld, 425.

4 Wichmann, Das \#hoffnungslicht aus Osterfeld, 425. 
Gleichzeitig gab es auch Pfarren, deren Pastoralteam für die Bevölkerung überhaupt nicht wahrnehmbar war und den Eindruck erweckte, sich nicht um „seine Schäfchen“ zu sorgen. Zahlreiche bis dahin höchst engagierte Pfarrmitglieder und treue Kirchgeher*innen fühlten sich in diesem Zusammenhang schwer verletzt.

Diese Aspekte spiegeln in unterschiedlicher Ausprägung zwei Seiten eines Kirchenbildes wider, in dem Hauptamtliche als Versorger- und Betreuer*innen der Gemeinde gesehen werden - zu oft wohl nicht zu Unrecht, ist es doch von den Hauptamtlichen über lange Jahre auch so gelebt worden - und als für die Gemeinde alleinig Zuständige. ${ }^{5}$ Die nächsten Jahre und Jahrzehnte werden zeigen, wie sich die Situation in den immer größer werdenden Räumen entwickeln wird - und wie an so vielen Stellen ist auch hier das Coronavirus wie eine Lupe, unter der manches deutlich zu Tage tritt und zu Veränderungen drängt.

Die Bilder aus dem Bereich der analogen Gottesdienste zeigen unterschiedliche Formen von Glaubensgemeinschaft. Angefangen mit zwei Bildern von Hausgottesdiensten ${ }^{6}$ : eines, in dem vernetze Gemeinschaft über analoge und zeitliche Räume hinweg verwirklicht wird, und ein zweites, welches stellvertretend steht für vielfältige Formen familiärer oder häuslicher Gebetsgemeinschaft. Das dritte Bild macht auf die Art und Weise des Feierns analoger Messen in Zeiten wechselnder staatlicher wie kirchlicher Corona-Verordnungen aufmerksam.

Bild I: Netzwerkgottesdienst in der Silvesternacht

Jahreswechsel 2020/21 - Übergang von einem Jahr voll von Unbekanntem, nie zuvor Erlebtem, Bedrängendem in ein neues Jahr. Der Gottesdienstvorschlag ${ }^{7}$ von www.netzwerk-gottesdienst.at liegt vor mir an meinem Lieblingsplatz, Kerzen brennen und ich bin dem Vorschlag gefolgt und habe Papier, Stift und den Kalender für 2021 bereitgelegt. Ein kleines Weihwasserfläschlein nahm ich letztens aus der Kirche mit, es lag zur Mitnahme im Weibwasserbecken. Ich frag mich: Wer mögen die anderen Menschen sein, die wie ich jetzt oder später oder früher daheimsitzen, allein oder mit anderen zusammen, und ebenfalls diesen Gottesdienst feiern? Ich denke an sie und beginne: Im Namen des Vaters und des Sohnes und des Heiligen Geistes. Amen.

5 Vgl. Brüske, Pluralisierungsschub im gottesdienstlichen Feiern, 422.

6 Zum Begriff Hausgottesdienst vgl. Hecht, www.Netzwerk-Gottesdienst.at, 434.

7 Vgl. Fritzl, Netzwerkgottesdienst zum Jahreswechsel. 
Netzwerk-Gottesdienst ist eine Initiative des Bereiches Bibel-Liturgie-Kirchenraum der Erzdiözese Wien. Unter diesem Projektnamen verbinden sich vier Dimensionen des Netzwerkes - Gottesdienst zu Hause feiern, Gottesdienstübertragungen, Füreinander beten und Netzwerk knüpfen. ${ }^{8}$ Unter „Gottesdienst zu Hause feiern" sind die Gottesdienstvorlagen eingereiht, mit denen Menschen zuhause Gottesdienst feiern können - alleine oder gemeinsam mit anderen analog vor Ort - und damit ein Netzwerk knüpfen mit anderen Personen, die ebenfalls mit diesem Feierblatt beten. Das Schema des Gottesdienstes, angelehnt an die Wort-Gottes-Feier, ist gleichbleibend - gefüllt wurden die einzelnen Abschnitte für die jeweiligen Sonn- und Festtage von Verfasser*innen aus unterschiedlichen Orten des deutschen Sprachraums. Aus den Netzwerk-Gottesdiensten heraus ist der im vierten Bild dargestellte digitale Gottesdienst entstanden.

\section{Bild II: Adventandacht in der Gasse}

Gegen Abend steht eine Familie am kleinen Platz zwischen den Häusern, andere Bewohner"innen der Gasse schauen aus ibren weit geöfneten Fenstern. Im Briefkasten haben sie alle in der Früh das Blatt mit den Texten und Liedern der heutigen Adventandacht gefunden. Es ist die letzte der vier Adventandachten, die sie miteinander feiern. Obwohl sie alle schon lange gemeinsam in dieser Gasse wohnen, beten sie in diesem Jahr zum ersten Mal miteinander. Sie singen und beten zusammen, hören Texte aus der Bibel, sprechen Fürbitten und teilen miteinander Lichtmomente.

Zusammentreffen mehrerer Haushalte im privaten oder öffentlichen Raum unterlag staatlichen Einschränkungen. Dieses Bild zeigt eine Weise der Verwirklichung von Gemeinschaft in dieser Zeit, in der - bei allen gleichzeitig sich ebenfalls ereignenden Konflikten - auch eine neue Nähe zwischen Nachbar*innen und auch Fremden zu spüren war. Basierend auf von Pfarren oder anderen Organisationen bereitgestellten Feierbehelfen sowie mithilfe des Gesangs- und Gebetsbuches „Gotteslob“ wurden gottesdienstliche Feiern auf kreative Weise gefeiert.

8 Sie alle sind auf der Homepage www.netzwerk-gottesdienst.at zu finden und bei Hecht, www.Netzwerk-Gottesdienst.at, näher beschrieben. 


\section{Bild III: Heilige Messe auf Abstand am Christtag}

Die Maßnahmen der Regierung besagen: Kein Volksgesang. Und das zu Weihnachten! So wird das Lied "Stille Nacht, heilige Nacht" einzig von der Kantorin vorgetragen. Das restliche musikalische Programm dieser heiligen Messe konzentriert sich, wie auch schon in den Wochen vorher und nachher, auf das Wesentliche - eine Strophe des Anfangsliedes, Halleluja und Sanctus. Volk und Priester schweigen die meiste Zeit beim Gesang, singen vielleicht im Herzen mit. Bei "Heilig" stimmt der Priester voll Freude ein. Vielleicht hat er in dem Moment einfach vergessen, dass er nicht singen „darf“. Die Kirche ist mäßig gefüllt - die allgemeinen Maßnahmen ${ }^{9}$ verlangten zu diesem Zeitpunkt einen Abstand von 1,5 Metern zwischen Menschen aus unterschiedlichen Haushalten. Konkretisierungen wurden von den einzelnen Diözesen/Pfarren verlautbart. ${ }^{10}$ Beim Friedensgruß schauen die Menschen sich gegenseitig an, nicken einander zu. Zur Kommunion drängen sie nach vorn - fast „wie früher".

Die miteinander in einem analogen Raum gefeierten Gottesdienste unterlagen in den ersten 18 Monaten den Bestimmungen der Maßnahmen zur Eindämmung der Ausbreitung von Covid-1911. So besagten die Rahmenordnungen je nach Pandemiephase in unterschiedlicher Intensität Maßnahmen bezüglich dem zu anderen Personen einzuhaltenden Abstand, der Zahl der Mitfeiernden, dem Tragen von Mund-Nasen-Schutz oder medizinischen Masken, dem Gesang und Gebet, den Worten beim Kommunionempfang und leiblichen Berührungen wie dem Friedensgruß. Das Verbot öffentlicher Messfeiern war ein harter Schlag für Menschen, für die der Empfang der Eucharistie ein Herz- und Lebensmittelpunkt ist. Für sie verwirklicht sich, was in Sacrosanctum Consilium (SC) geschrieben steht: „Aus der Liturgie, besonders aus der Eucharistie, fließt uns wie aus einer Quelle die Gnade zu“ (SC 10). Andere gottesdienstliche Feiern oder das Mitfeiern einer digitalen Messfeier waren da zwar möglich, erfüllen aber auf Dauer nicht die Sehnsucht nach dem Empfang der heiligen Eucharistie.

Die Maßnahmen lenkten jedoch auch das Augenmerk auf bisher Selbstverständliches und vielleicht auch mitunter kaum Wahrgenommenes: Es

9 Vgl. Österreichische Bischofskonferenz, Rahmenordnung der Österreichischen Bischofskonferenz zur Feier öffentlicher Gottesdienste (wirksam vom 7. Dezember 2020 bis vorerst 6. Jänner 2021).

10 Vgl. Kathpress, Kirchen stellen Regeln für „sichere“ Weihnachtsgottesdienste auf.

11 Vgl. Österreichische Bischofskonferenz, Behelfe / Handreichungen. Rahmenordnung \& Präventionskonzept der Österreichischen Bischofskonferenz zur Feier der Gottesdienste. 
ist die Aufmerksamkeit auf die Verwirklichung der tätigen Teilnahme aller Gläubigen an gottesdienstlichen Feiern, die nun nur eingeschränkt möglich war. Dies betrifft zunächst die Bedeutung von ausgesprochenen und hörbaren Worten, wenn Gebete nicht laut gebetet werden können und der Kommunionempfang schweigend vonstattengeht. Sodann kommt die Bedeutung von Musik in den Blick - sowohl der selbst gesungenen als auch der gehörten. Die Kirchenmusiker*innen, Kantor*innen und andere in den Gottesdiensten Musizierenden haben auch stellvertretend für das ganze Volk Herzenstöne ausgedrückt, Emotionen in Worte verwandelt. Singen und hörbar miteinander Beten verbindet Menschen auf einer anderen Ebene, als körperliche Co-Präsenz es tut. Und schließlich hat auch der Friedensgruß durch die notwendige Veränderung der Form wieder neue Aufmerksamkeit erhalten. Reichte man sich sonst, verbunden mit den Worten „Der Friede sei mit Dir“, die Hand - wobei die Augen oftmals schon zur nächsten Person wanderten, wurde es nun unabdingbar, die andere Person wirklich anzuschauen, um einander (zeitweise sogar wortlos) zunickend den Frieden zu wünschen. Diese neue Aufmerksamkeit des bewussten Anschauens der Mitmenschen, des bewussten Augenblickes kann als Vertiefung der diakonischen Dimension der Liturgie gesehen werden.

\subsection{Digitale Gottesdienste}

Gottesdienstübertragung und „religiös motiviertes Handeln via Internet“12 sind neben den analogen Feiern das zweite Feld der Bilder gottesdienstlicher Feiern der Corona-Zeit, die hier dargestellt werden. Vor bald 90 Jahren, zu Weihnachten 1931, fand mit Papst Pius XI. die erste Übertragung eines Gottesdienstes im Radio statt. Damit begann die Geschichte der Radiogottesdienste und $\mathrm{ab}$ dem Ende der fünfziger Jahre auch der Fernsehgottesdienste. Hervorzuheben sind für den deutschen Sprachraum die Übertragungen Evangelischer Gottesdienste und Katholischer Messen, die seit Mitte der achtziger Jahre abwechselnd wöchentlich im ZDF gesehen und mitgefeiert werden können. Zusätzlich werden auch zu hohen Feiertagen Gottesdienste gesendet. ${ }^{13}$ Teresa Berger spannt den Bogen weiter zurück zu den Anfängen der Liturgie und betont: „,...] digital-mediatisierte liturgische Praxis [steht] in Kontinuität zu früheren Vollzügen. Es

12 Jeggle-Merz, Gottesdienst und mediale Übertragung, 463.

13 Vgl. zur Geschichte der Übertragungen von Gottesdiensten: Jeggle-Merz, Gottesdienst und mediale Übertragung, 467-470. 
gibt letztlich keine un-mittelbaren, nicht mediatisierten gottesdienstlichen Vollzüge, sondern immer nur den Zusammenfluss jeweils verfügbarer Medien, wie zum Beispiel Körper, Klänge, Gewand oder Architektur. Digitalmediatisierte Gottesdienstpraxis heute ist deshalb im Kontext der gesamten Liturgiegeschichte zu sehen"14. Ihr ist es wichtig "die Unterschiede zwischen digitaler und prädigitaler Praxis nicht überzubetonen. " 15 Auch der Unterschied im Bezug auf diese Feiern zwischen der Zeit vor Corona und mit Corona soll hier nicht überbetont werden. Die einzelnen Elemente, sowohl im analogen als auch im digitalen Bereich, gab es ja zu einem großen Teil bereits früher.

Worin äußert sich, dass eine Person, die sich zu einem digitalen Gottesdienst zuschaltet, Rezipientin oder Zuschauerin/Zuhörerin ist? Diese Frage ist auch in Bezug zu analogen Gottesdiensten zu stellen. Es bedarf jedoch bei digitalen Feiern, die in einem virtuellen Raum gefeiert oder die über einen Bildschirm/das Radio angesehen oder angehört werden, einer körperlichen Fokussierung und einem Mehr an innerer Konzentration. Was im analogen Gottesdienstraum vielen Gläubigen durch den gemeinsamen Vollzug selbstverständlich ist, muss hier bewusst allein vollzogen werden. Rezipientin einer Feier wird eine zuschauende/zuhörende Person durch ein Mehr an Teilnahme. „Dieses ,Mehr an Teilnahme' drückt sich häufig in einem spezifischen Verhalten während der Übertragung aus (z. B. Entzünden einer Kerze, Bereitlegen und Gebrauch des Gesangbuchs, Mitvollzug der Körperhaltungen). " ${ }^{16}$ Das sechste Bild ist dafür ein Beispiel.

Die im sechsten Bild angerissene Situation ist auch ein Beispiel für die Form der Gemeinschaft, die über digitale gottesdienstliche Feiern möglich ist. So ist hier "die ,empfundene' Gemeinschaft entscheidend, nicht die ,physische" Gemeinschaft an ein und demselben Ort. "17 Von unterschiedlichen Orten, Städten und Ländern her schalten sich Menschen zusammen. Sie nehmen in einem Zoom-Raum an einer gemeinsamen Feier teil ${ }^{18}$ und drücken so Verbundenheit über Grenzen und Meere hinweg sichtbar und hörbar aus. Oder sie nehmen an einem im Fernsehen oder im Internet übertragenen Gottesdienst teil und tauschen sich im Anschluss darüber aus. Dazu dienen auch die am Ende von Fernsehgottesdiensten eingeblendeten Telefonnummern. Auf diese Weise wird spürbar: „Die

14 Berger, Liturgie digital, 263.

15 Berger, Liturgie digital, 263.

16 Jeggle-Merz, Gottesdienst und mediale Übertragung, 463.

17 Berger, Liturgie digital, 264.

18 Fritzl, Lasst uns zoomen!, berichtet von einer Zoom-Feier mit der Angehörigen der brasilianischen Partnergemeinde, die Weltkirche greifbar macht. 
Gemeinde Jesu ist ein Netzwerk [...ein] virtuelles Netzwerk"19. Auf der Ebene der Weltkirche wird dies besonders bei gottesdienstlichen Feiern mit dem Papst deutlich, im Jahr 2020 etwa beim außerordentlichen Segen Urbi et Orbi am 27.03.2020, der von dokumentarisch Interessierten auch jetzt noch angeschaut werden kann. ${ }^{20}$ Die auf der Seite verzeichnete Zahl von 19.819 (Stand: 13.08.2021) Zuseher*innen muss im Kontext dessen gesehen werden, dass die Feier von unzähligen Fernsehanstalten live übertragen wurde und mitunter auch dort noch nachgeschaut werden kann ${ }^{21}$. Charakteristisch für digitale gottesdienstliche Feiern ist die Öffentlichkeit. Der offene Zugang ist bei Feiern via TV, YouTube, Facebook zumeist gegeben. Mitunter sind jedoch eine Registrierung oder die Anforderung eines Passwortes notwendig, um an Feiern teilzunehmen. Der Zugang zu Feiern im Internet erfordert weiters auch eine stabile Internetverbindung.

Mit den vier Bildern digitaler Gottesdienste sind auch Annäherungen an die Thematik der Körperlichkeit verbunden. „Im virtuellen Raum gibt es über das Hören und Sehen hinaus keine gemeinsamen sinnlichen Erfahrungen (Riechen, Schmecken, Berühren), wenn sie nicht außerhalb der medialen Kommunikation , analog ${ }^{6}$ herbeigeführt werden, z. B. durch das Verbrennen von Weihrauch, das Essen und Trinken bei einer Agapefeier am häuslichen Tisch oder das Kreuzzeichen, mit dem sich Ehepaare oder Familien am Ende einer Feier gegenseitig segnen."22 Man kann die anderen Teilnehmer*innen einer Feier nicht spüren - nur sehen, manche hören und manchmal auch von ihnen lesen. Digitale Feiern blenden die leibliche Dimension des Menschen nicht aus, sie wird jedoch anders vollzogen, als bei analogen Feiern. Bei der Gestaltung gilt es immer wieder, diese Dimension bewusst mit einzubeziehen und digitale und analoge Elemente in den Ablauf des Gottesdienstes zu integrieren, denn „durch sinnenfällige Zeichen wird in ihr die Heiligung des Menschen bezeichnet und in je eigener Weise bewirkt und vom mystischen Leib Jesu Christi, d. h. dem Haupt und den Gliedern, der gesamte öffentliche Kult vollzogen.“ (SC 7).

19 Wiefel-Jenner, Betet heute jemand die \#twomplet vor?, 95.

20 Vgl. EWTN, Papst Franziskus spendet den Segen „Urbi et Orbi“, 27.03.2020.

21 Beispielsweise über den YouTube-Kanal von ZDFheute Nachrichten. Dort sind es 123.034 Aufrufe (Stand 13.08.2021), siehe: ZDFheute Nachrichten: Corona-Urbi et Orbi: Papst spendet Sondersegen.

22 Brüske, Pluralisierungsschub im gottesdienstlichen Feiern, 420. 


\subsubsection{Gottesdienstliche Feiern gemeinsam in einem "digitalen Raum"}

Unter gottesdienstlichen Feiern. die in einem gemeinsamen digitalen Raum stattfinden, zeige ich das Bild einer Karfreitagsfeier im Zoom-Raum sowie eines Mittagsgebets in einer WhatsApp-Gruppe. Es sind zwei Beispiele von vielen Feierformen, die in dieser Corona-Zeit entstanden sind und teilweise auch zu Zeiten entwickelt wurden, in denen es durchaus möglich war, sich wieder analog zu treffen. So hörte ich von einer Initiative, durch die Fastenzeit 2021 hindurch bis Pfingsten jeden Abend um 21 Uhr gemeinsam die Komplet zu beten. ${ }^{23}$ Eine andere bietet unter dem Namen \#ZusammenHALT jeweils am 15. und 30. des Monats ein Abendgebet an. ${ }^{24}$ An jedem vierten Sonntag im Monat wird in der Diözese Graz-Seckau um 18 Uhr via Zoom zur Online-Feierstunde mit dem Namen FEIERabend eingeladen. Unterschiedliche Personen gestalten hier die einzelnen Abende und geben so auch einen Einblick in die Vielfalt der Diözese. ${ }^{25}$

\section{Bild IV: Karfreitagsgottesdienst im Zoom-Raum}

Karfreitag 2021, 18:15 Uhr. Das Vorbereitungsteam, die Technikerin, die Kantor"innen und die Lektorinnen treffen sich im Zoom-Raum. "In Wirklichkeit" sitzen sie an unterschiedlichen Orten in Österreich und Deutschland. Der Gottesdienst wird Schritt für Schritt nochmal durchgegangen, Lektorinnen und Kantor"innen proben - dann kann's los gehen. Um 18:45 wird der Zoom-Raum für alle geöffnet, die an diesem Abend hier zusammen des Leidens und Sterbens Jesu Christi gedenken möchten. Eine Person nach der anderen betritt den Raum, wird begrüßt. Um 19 Uhr wird der Teaser gezeigt. Während der Feier werden Bilder eingeblendet und ebenso die Lieder, die alle, bei stumm geschaltetem Mikrophon, mit den Kantor*innen zusammen singen können. Jeder und jede Teilnehmer*in ist eingeladen, Fürbitten in den Chat zu schreiben. Zum Abschluss wird zur persönlichen Betrachtung ein Kreuzbild eingeblendet, begleitet von Musik aus Taizé.

Ein Team von drei Grazer Theolog*innen gestaltete die drei heiligen Tage Gründonnerstag, Karfreitag und Osternacht, die liturgisch als die eine Feier des Leidens, Sterbens und der Auferstehung Christi betrachtet werden, sowie einen Abendgottesdienst am Ostersonntag, basierend auf den über

23 Vgl. Fritzl, Lasst uns zoomen!

24 Bistum Limburg Bischöfliches Ordinariat, \#ZusammenHALT.

25 Katholische Kirche Steiermark, FEIERabend. 
Netzwerk-Gottesdienst.at ${ }^{26}$ abrufbaren Feiervorlagen und so mit diesen in deutlicher Verbindung stehend, und lud dazu ein, gemeinsam diese gottesdienstlichen Feiern zu begehen. Der Link war auf der Seite der Diözese Graz-Seckau abrufbar, eine Anmeldung oder die Eingabe eines Passworts waren nicht notwendig.

$\mathrm{Zu}$ einer Zeit, in der der Volksgesang in der Kirche nicht gestattet war, konnten die Menschen sich am Gesang beteiligen und auch im Schreiben, Schweigen und Teilen von Gedanken Gemeinschaftserfahrungen machen. Ihre Alltagswelt wurde zum Feierraum. Dies kann „mit einer Veränderung dieser Alltagswelten einhergehen, manifest durch religiöse Zeichen und auch geistlich, was allerdings kein neues Phänomen ist, wenn man z. B. an Andachtsecken denkt." ${ }^{27}$

Die Teilnehmer*innen kamen aus dem ganzen deutschen Sprachraum, manche kamen täglich, andere nahmen einmalig die Gelegenheit zur Teilnahme wahr. Sie konnten sich in selbst gewählter Möglichkeit der Anonymität aktiv tätig einbringen oder auch zurückziehen.

\section{Bild V: Mittagsgebet via WhatsApp}

Pling. Pling. Pling. Nacheinander treten die Teilnehmerinnen und Teilnehmer einer Online-Tagung ${ }^{28}$ der WhatsApp-Gruppe bei, in welcher das gemeinsame Mittagsgebet stattfinden wird. Am Beginn steht die Einladung, während des Glockenläutens, welches via Audiodatei zu hören ist, eine bereitgestellte Kerze $z u$ entzünden und ein Foto dieser Kerze in den Chat zu stellen. Nach und nach werden Texte, ein Video und weitere Audiodateien zum gemeinsamen Beten, Singen, Meditieren in den Chat gestellt und auch dazu eingeladen, Anliegen oder Namen der Menschen, für die man gerade betet, zu schreiben. Mit dem gesprochenen oder gesungenen "Singet Lob und Preis. Dank sei Gott dem Herr" “ endet das Gebet.

Ähnlich wie dieses Mittagsgebet läuft auch die seit 2014 bei Twitter stattfindende \#twomplet ab. Twomplet ist ein Kofferwort, zusammengesetzt aus Twitter und Komplet, und ist der Name eines überkonfessionellen Abendgebetes, welches in struktureller Anlehnung an die Komplet um 21

26 Vgl. Bild I.

27 Brüske, Pluralisierungsschub im gottesdienstlichen Feiern, 420.

28 „Liturgie im Gespräch 2021 Spezial: Liturgie und Corona“, veranstaltet vom Liturgischen Institut der Schweiz am 23.02.2021 via Zoom, weitere Informationen unter: Liturgie im Gespräch 2021 Spezial: Liturgie und Corona. 
Uhr via Twitter Menschen zum Gebet versammelt. ${ }^{29}$ Es ist eine schlichte Form des gemeinsamen Gebetes, in dem die Konzentration zunächst voll auf sichtbaren Worten liegt. Gemeinschaft entsteht im Sehen der Worte und Bilder der Anderen - der Namen, Fotos, geteilten Gebetsanliegen, die im Lesen ein zweites Mal verbalisiert werden.

\subsubsection{Gottesdienstübertragungen}

Im Bereich der Gottesdienstübertragungen weisen die folgenden Ausführungen einerseits auf Fernsehgottesdienste aus dem ZDF hin und andererseits auf Gottesdienste, die über diverse Kanäle im Livestream mitgefeiert werden können. Diese Übertragungen waren besonders in den ersten Monaten der Corona-Pandemie für viele Menschen der zentrale Ort, an dem sie, von ihren gewohnten Alltagsräumen aus, Liturgie feiern konnten. Für die Produzent*innen von Übertragungen und Livestreams bedeutet dies auch eine hohe Verantwortung und einen großen Zeitaufwand. Vielfältige Hinweise für die Fernsehgottesdienste sind in der 2007 in zweiter Auflage erschienenen Arbeitshilfe Fernsehgottesdienst der Deutschen Bischofskonferenz zusammengetragen. ${ }^{30}$ Dieses Dokument ist die Überarbeitung einer Arbeitshilfe aus dem Jahr 1989. Speziell für Personen, die bisher im Bereich von Streaming noch wenig oder keine Erfahrungen gesammelt hatten, wurden Tipps und Hilfen zusammengetragen und ins Internet gestellt. ${ }^{31}$ Gottesdienstübertragungen sind immer hybride Formate. Die vor Ort, analog im Raum, Feiernden (so klein ihre Zahl auch sein mag) bilden mit den über die diversen Medien zugeschalteten Personen eine Gottesdienstgemeinde. Verstärkt hat sich im Vergleich zu früheren Fernseh- oder auch über andere Plattformen gestreamten Gottesdiensten dabei der Fokus auf die vor den Bildschirmen versammelten Rezipient*innen und Zuschauer*innen der Feier. Das gezeigte Bild und der Ton sind von

29 Vgl. Wiefel-Jenner, Betet heute jemand die \#twomplet vor?

30 Sekretariat der Deutschen Bischofskonferenz, Gottesdienst-Übertragungen; vgl. auch Fischer, Mystagogische Bildregie, als eine weitere Arbeitshilfe für Regisseure/innen, TV-Redakteure/innen und Medienverantwortliche der Kirche, herausgegeben von der Katholische Fernseharbeit Arbeitsstelle der Deutschen Bischofskonferenz.

31 Vgl. Sindelar, Martin: Gottesdienste im Netz teilen. Liturgie im Live-Streaming; Neumann, Felix: Die besten Tipps für Livestreams von Gottesdiensten. Diese Zusammenstellung stammt vom 1. April 2020. 
immenser Bedeutung. Beides vermittelt die Botschaft von Kirche und des Kirchenbildes.

\section{Bild VI: Fernsehgottesdienst aus Bensheim im ZDF}

Juni 2020, am Sonntagnachmittag ein Telefonat mit der Großmutter.

$S:$,Und, hast Du heut Vormittag wieder die Messe im ZDF angeguckt?"32

A: "Ich sage: mitfeiern. Und ja, ich habe die Messe wieder mitgefeiert. Ich zünde eine Kerze an. Neben mir auf dem Stubl liegt das Gotteslob, ich singe mit, bin innerlich ganz dabei. Es war wieder der Pfarrer Förg mit einer Gruppe junger Leute, die gesungen haben. Sie singen so schön!"

S: "Ja, ich habe auch nochmal nachgeguckt. Schon drei Mal waren sie dran. Es ist ein Vokalensemble, das da miteinander singt. Und der Pfarrer spricht die Menschen direkt an - die wenigen, die mit ihm in der Kirche sein können und die vielen, die von zuhause aus zugucken, ähm mitfeiern."

In diesem Bild sind Eindrücke im Gefolge eines Fernsehgottesdienstes eingefangen. Es war besonders in diesem Corona-Jahr, dass zwischen dem 22. März 2020 und dem 9. Mai 2021 der Gottesdienst sieben Mal (am 22. März 2020, 19. April 2020, 31. Mai 2020, 14. Juni 2020, 17. Januar 2021, 25. April 2021 und 9. Mai 2021) aus Bensheim übertragen wurde. Jedes Mal sangen dabei Mitglieder des Jungen Vokalensembles St. Georg, vier Mal stand Pfarrer Heinz-Jürgen Förg der Liturgie vor, die ersten vier Gottesdienste wurden aus der Hospitalkirche St. Joseph in Bensheim übertragen. ${ }^{33}$ Auf diese Weise entstand Verbundenheit zu einem Ort und zu konkreten Personen (dem Vokalensemble und Pfarrer Förg), die das Gefühl, eine Gemeinschaft in dieser schweren Zeit zu sein, verstärkte.

Bild VII: „Charme des Lokalen“34_ Gottesdienste im Livestream über Facebook, YouTube oder eigene Webseiten

Palmsonntag 2020. „Mess-hopping“. Live dabei zunächst bei der Messe um 9 Uhr aus einer Klosterkirche. Es ist die erste Übertragung von dort, die Priester kenn ich und es ist gleich eine Verbundenheit spürbar. Leider funktioniert der Ton nicht, so ist es sehr mühsam. Also weiter - um 9:30 zum ZDF Fernsehgottesdienst aus dem Wiener Stephansdom. Hier funktionieren Bild und Ton in

32 14.06.2020 (Pfarrer Heinz-Jürgen Förg und Junges Vokalensemble St. Georg, Hospitalkirche St. Joseph Bensheim (Bistum Mainz)); Vgl. Kirche.tv, Hospitalkirche St. Joseph, Bensheim. Katholischer Gottesdienst.

33 Vgl. ZDF, Gottesdienst Jahrespläne.

34 Brüske, Pluralisierungsschub im gottesdienstlichen Feiern, 408. 
bewährter Qualität, ich feiere diese Messe mit. Anschließend schau ich noch auf Facebook in der Pfarre einer befreunden Kantorin vorbei. Sie sind schon am Ende der Messe angekommen, durch die Kamera ist von schräg unten der Altarraum zu sehen. Da die Sonne gerade durch die Fenster direkt auf die Kamera $z u$ scheint, sieht man leider die Personen nur schwer - aber es ist berührend, Menschen zu sehen, die ich kenne und mit ibnen zusammen zu beten. Am Abend bin ich neugierig, wie im Petersdom der Palmsonntag gefeiert wurde und schau bei YouTube nach und ein Stück in den Gottesdienst hinein.

„Die eigene Kirche, den Heimatpfarrer zu sehen, kann schon ein Wert an sich sein." ${ }^{35}$

Bereits im sechsten Bild wurde der Wert des Vertrauten angesprochen. Ebensolches gilt auch im Blick auf Gottesdienste via Livestream. Durch die Übertragungen ist es möglich, Gottesdiensten und Gebetszeiten an vertrauten Orten (der Heimatgemeinde oder einem anderen Ort, zu dem ein besonderer Bezug besteht, wie etwa Taizé) mitzufeiern. Diese Feiern haben ebenso wie die Fernsehgottesdienste öffentlichen Charakter. Je nach Voreinstellungen der Seitenbetreiber"innen ist eine Livekommunikation im Chat mit Mitfeiernden möglich. Die tätige Teilnahme kann sich auch darauf erstrecken, Fürbitten einzusenden, die dann in der Feier vorgelesen oder anders präsent gemacht werden. ${ }^{36}$ Trauer und Angst, Hoffnungen und Bitten werden so gemeinsam vor Gott getragen.

Einige via Livestream gesendeten Gottesdienste sind nur live zu sehen, andere sind auch später noch abrufbar und haben dann dokumentarischen Charakter. Die Gottesdienste sind von unterschiedlicher Qualität - zu einem großen Teil sind ehrenamtlich tätige Menschen die Produzent*innen der Livestreams, technisch erfahrene aber auch sich ganz neu einarbeitende Personen, die Bildregie und Technik überhaupt erst erlernen. Andere Pfarren (oder auch Klöster) senden schon länger live ihre Gottesdienste. So beispielsweise die Pfarre Hartberg in der Steiermark. Seit 2008 wird der sogenannte IGOD von dort via YouTube ${ }^{37}$ in alle Welt gesendet. ${ }^{38}$ Pfarrer Reisenhofer formuliert die Erwartungshaltung so: „Wenn irgendwo auf der Erde ein Mensch durch den Internetgottesdienst ein kleines Licht der Hoffnung für sein Leben entdeckt, hat sich die Mühe gelohnt. “" ${ }^{\text {(39 }}$

35 Neumann, Die besten Tipps für Livestreams von Gottesdiensten.

36 Vgl. z.B. Missio, Gottesdienst Livestream. Angebote und Hilfestellungen.

37 IGOD Pfarre Hartberg.

38 Pfarre Hartberg, Internetgottesdienste Pfarre Hartberg.

39 Reisenhofer, Zur Erwartungshaltung. 


\section{Abschließende Notizen}

Verbundenheit und Ferne, Chancen und Grenzen, Entwicklungen, Einschränkungen und Möglichkeiten - die vorgestellten sieben Bilder gottesdienstlicher Feiern während unterschiedlicher Phasen der Corona-Pandemie geben Einblicke in das öffentliche und private Glaubensleben von Menschen. Die nächsten Jahre werden zeigen, wie es mit den Aufbrüchen und neu eröffneten Möglichkeiten weitergehen wird. Vom Livestream zum Gassengottesdienst - welche Elemente werden weitergetragen? Welche digitalen Formate werden sich auf längere Zeit bewähren? Welche anderen Prozesse werden innerhalb der Pastoral und Liturgie, in der Kirche und in den theologischen Wissenschaften in Gang gesetzt?

\section{Literatur und Internetseiten}

Berger, Teresa: Liturgie digital. Zu gottesdienstlichen Vollzügen in Bits \& Bytes, in: Liturgisches Jahrbuch 69 (2019), 253-268.

Bistum Limburg Bischöfliches Ordinariat: \#ZusammenHALT. Digital verbunden Gottesdienste feiern, in: https://meditationszentrum.bistumlimburg.de/beitrag/z usammenhalt-3/ [11.08.2021].

Brüske, Gunda: Pluralisierungsschub im gottesdienstlichen Feiern. Beobachtungen in der Zeit der Corona-Pandemie aus der deutschsprachigen Schweiz, in: Bukovec, Predrag/Volgger, Ewald (Hg.): Liturgie und Covid-19. Erfahrungen und Problematisierungen, Regensburg: Pustet 2021 (= Schriften der Katholischen Privat-Universität Linz 11), 401-423.

EWTN - Katholisches TV: Papst Franziskus spendet den Segen „Urbi et Orbi“. 27.03.2020, in: https://www.youtube.com/watch?v=SIMvoAhFRaE [13.08.2021].

Fischer, Ulrich: Mystagogische Bildregie in Fernsehgottesdiensten. Arbeitshilfe für Regisseure/innen und TV-Redakteure/innen und Medienverantwortliche der Kirche. Herausgegeben von der Katholische Fernseharbeit Arbeitsstelle der Deutschen Bischofskonferenz, Frankfurt am Main ${ }^{5} 2020$, in: https://fernsehen .katholisch.de/Portals/0/PDF/MystagoischeBildregie2020.pdf?ver=2020-04-03-09 3524-600 [19.08.2021].

Fritzl, Elisabeth: Lasst uns zoomen! Feiern im digitalen Raum, in: https://www.fein schwarz.net/lasst-uns-zoomen-feiern-im-digitalen-raum/ [10.08.2021].

Fritzl, Elisabeth: Netzwerkgottesdienst zum Jahreswechsel. 31.12.2020, in: https://w ww.netzwerk-gottesdienst.at/dl/nmpKJKJnOnmOJqx4NJK/netzwerk-gottesdiens t_Silvester_31122020_pdf [10.08.2021].

Gaudium et Spes (GS) wird zitiert nach: Pastorale Konstitution Gaudium et Spes. Über die Kirche in der Welt von heute, in: https://www.vatican.va/archive/hist_c ouncils/ii_vatican_council/documents/vat-ii_const_19651207_gaudium-et-spes_ ge.html [13.08.2021]. 
Hecht, Pia: www.Netzwerk-Gottesdienst.at. Erfahrungsbericht einer vernetzenden Corona-Initiative online, in: Bukovec, Predrag/Volgger, Ewald (Hg.): Liturgie und Covid-19. Erfahrungen und Problematisierungen, Regensburg: Pustet 2021 (= Schriften der Katholischen Privat-Universität Linz 11), 429-441.

IGOD Pfarre Hartberg: https://www.youtube.com/channel/UCh9ugFaLi_KGD3Ep Q1fF7lQ [12.08.2021].

Jeggle-Merz, Birgit: Gottesdienst und mediale Übertragung, in: Häußling, Angelus A./Klöckener, Martin/Messner, Reinhard (Hg.): Gottesdienst der Kirche. Handbuch der Liturgiewissenschaft. Band 2: Theologie des Gottesdienstes, Regensburg: Pustet 2008, 455-487.

Katholische Kirche Steiermark: FEIERabend. Online-Feierstunde jeden vierten Sonntag im Monat um 18 Uhr via ZOOM, in: https://www.katholische-kirc he-steiermark.at/portal/glaubenfeiern/glaubenfeiern/gottesdienstfeiern/artikelgo ttesdienst/article/25945.html [11.08.2021].

Kathpress: Kirchen stellen Regeln für „sichere“ Weihnachtsgottesdienste auf, in: https://www.bischofskonferenz.at/132501/kirchen-stellen-regeln-fuer-sichere-wei hnachtsgottesdienste-auf [09.08.2021].

Kirche.tv: Hospitalkirche St. Joseph, Bensheim. Katholischer Gottesdienst, in: https://fernsehen.katholisch.de/fernsehgottesdienste/jahresplan2020/zdfgott esdienst20200614 [20.07.2021].

Liturgie im Gespräch 2021 Spezial: Liturgie und Corona, in: https:/www.liturgie.c h/kurse/liturgie-im-gespraech/liturgie-im-gespraech-vergangene-tagungen/liturgi e-im-gespraech-spezial [10.08.2021].

Missio: Gottesdienst Livestream. Angebote und Hilfestellungen, in: https:/www.m issio.at/gottesdienst-livestream-heilige-messe/ [09.08.2021].

Netzwerk Gottesdienst: https://www.netzwerk-gottesdienst.at/ [09.08.2021].

Neumann, Felix: Die besten Tipps für Livestreams von Gottesdiensten. So gelingt das gemeinsame Online-Gebet in der Corona-Krise, in: https://www.katholi sch.de/artikel/25011-die-besten-tipps-fuer-livestreams-von-gottesdiensten [10.08.2021].

Österreichische Bischofskonferenz: Behelfe/Handreichungen. Rahmenordnung \& Präventionskonzept der Österreichischen Bischofskonferenz zur Feier der Gottesdienste, in: https://www.bischofskonferenz.at/behelfe/corona-rahmenordnun g-und-praeventionskonzept [09.08.2021].

Österreichische Bischofskonferenz: Rahmenordnung der Österreichischen Bischofskonferenz zur Feier öffentlicher Gottesdienste (wirksam vom 7. Dezember 2020 bis vorerst 6. Jänner 2021), in: https://www.bischofskonferenz.at/dl/mt MkJmoJKmkNJqx4KJKJKJKLLooN/Rahmenordnung_der_BK_zur_Feier_ffe ntlicher_Gottesdienste_03122020_pdf[09.08.2021].

Pfarre Hartberg: Internetgottesdienst, in: https://hartberg.graz-seckau.at/pfarre/618 3/internetgottesdienst/wassindinternetgottesdien [09.08.2021].

Reisenhofer, Josef: Zur Erwartungshaltung, in: https://hartberg.graz-seckau.at/pfarr e/6183/internetgottesdienst/videoteam [12.08.2021]. 
Sacrosanctum Concilium (SC) wird zitiert nach: Konstitution über die heilige Liturgie. Sacrosanctum Concilium, in: https://www.vatican.va/archive/hist_cou ncils/ii_vatican_council/documents/vat-ii_const_19631204_sacrosanctum-concil ium_ge.html [13.08.2021].

Sekretariat der Deutschen Bischofskonferenz (Hg.): Gottesdienst-Übertragungen in Hörfunk und Fernsehen Leitlinien und Empfehlungen 2002. Aktualisierte, überarbeitete und erweiterte Fassung der „Leitlinien für die mediale Übertragung von gottesdienstlichen Feiern“ (= Arbeitshilfen 72, 1989), Bonn ${ }^{2} 2007$ (= Arbeitshilfen 169).

Sindelar, Martin: Gottesdienste im Netz teilen. Liturgie im Live-Streaming, in: https://iturgie.ch/images/liturgie/praxis/corona/Liturgie_im_Livestream_Leitlin ien.pdf [23.07.2021].

Wichmann, Christoph: Das \#hoffnungslicht aus Osterfeld. Eine Kerze im Fenster geht viral, in: Bukovec, Pedrag/Volgger, Ewald (Hg.): Liturgie und Covid-19. Erfahrungen und Problematisierungen, Regensburg: Pustet 2021 (= Schriften der Katholischen Privat-Universität Linz 11), 424-428.

Wiefel-Jenner, Katharina: ,Betet heute jemand die \#twomplet vor?‘, in: Deeg, Alexander/Lehnert, Christian (Hg.): Liturgie - Körper - Medien. Herausforderungen für den Gottesdienst in der digitalen Gesellschaft, Leipzig: Evangelische Verlagsanstalt 2019 (= Beiträge zu Liturgie und Spiritualität 32), 95-112.

ZDF: Gottesdienst Jahrespläne, in: https://www.zdf.fernsehgottesdienst.de/jahrespl aene [20.07.2021].

ZDFheute Nachrichten: Corona-Urbi et Orbi. Papst spendet Sondersegen, in: https:/www.youtube.com/watch?v=EKqnA5WRh-E [13.08.2021]. 
